\title{
Tolosa Hunt Syndrome: Current Diagnostic Challenges and Treatment
}

\author{
Samwel Sylvester Msigwa*, Yan Li, Xianglin Cheng \\ Department of Neurology, The Clinical Medicine School of Yangtze University, The First Affiliated Hospital of Yangtze \\ University, Jingzhou, China \\ Email: ^1775966639@qq.com
}

How to cite this paper: Msigwa, S.S., Li, Y. and Cheng, X.L. (2020) Tolosa Hunt Syndrome: Current Diagnostic Challenges and Treatment. Yangtze Medicine, 4, 140-156. https://doi.org/10.4236/ym.2020.42014

Received: August 27, 2019

Accepted: June 26, 2020

Published: June 29, 2020

Copyright $\odot 2020$ by author(s) and Scientific Research Publishing Inc. This work is licensed under the Creative Commons Attribution International License (CC BY 4.0).

http://creativecommons.org/licenses/by/4.0/

\begin{abstract}
Tolosa-Hunt syndrome (THS) is an uncommon diagnosis with an incidence of nearly 1 to 2 cases per million hallmarked by the presence of painful ophthalmoplegia (PO) due to a granulomatous inflammation (GI). Diagnostically, the major THS challenges encountered are owing to the exclusion of other GI presenting conditions necessitating multi-specialization consultations. This article presents uniquely advances in diagnosis and challenges encountered attempting to exclude THS mimics, details on physical examination and laboratory investigations have been incorporated. Tolosa Hunt MRI protocol (contrast-enhanced MRI), restricted diffusion and CISS MRI have lately proved to be precise investigations for THS diagnosis and follow up, on the contrary, number of false-negative/positive MRI diagnoses appears to be rising, hence proposed that MRI or biopsy shouldn't be mandatory criteria for diagnosis as opposed to IHS 2018 guidelines. Despite corticosteroids being the cornerstone therapy for THS, there are controversies concerning the better administration route, optimal dosage, and therapy longevity, through case reports, high dose IV mPSL of $500 \mathrm{mg}$ BID for 3 days, followed with maintenance dose of prednisone $60 \mathrm{mg}$ resulted in the earliest recovery, hereafter standardized guidelines are required. Alternatively, infliximab (300 mg infusion), azathioprine, methotrexate, and acupuncture can be used, farther studies are required to appraise the effectiveness and their safety. On the other side, microsurgery can be utilized for GI evacuation however associated risks of permanent CN VI palsy have been stated, nonetheless, GKRS can be employed when contraindication or corticosteroid intolerability exists though the hazard of late malignancy is a drawback.
\end{abstract}

\section{Keywords}

Tolosa-Huntsyndrome, Painfulophthalmoplegia, Methylprednisolone (mPSL), Gamma Knife Radiosurgery (GKRS), Constructive Interference in Steady-State (CISS) 


\section{Introduction}

Headache Classification Committee of the International Headache Society (IHS) in 2018 defined Tolosa-Hunt syndrome (THS) as one-sided orbital or periorbital painfulness associated with partial paralysis or weakness of either oculomotor, trochlea and/or abducens cranial nerves(CN) due to a granulomatous inflammation (GI) in the cavernous sinus (CS), superior orbital fissure (SOF) or orbit moreover recently there are reported incidences on the trigeminal nerve (ophthalmic), facial nerve and CN VIII [1]. THS is an unusual pathology listed by the National Organization for Rare Disorders (NORD) as an uncommon disorder defined by severe periorbital cephalalgia accompanied with ophthalmoplegia [2] [3].

Historically THS can be tracked back to 65 years ago where a neurosurgeon from Spain, Dr. Eduardo Tolosa paved a way into THS by elucidation of the clinical and pathological presentation of the syndrome. In 1961, seven years following Dr. Tolosa publication, Hunt et al. brought 6 new patients with similar findings, therefore, came into a conclusion that painful ophthalmoplegia (PO) was due to the CS inflammatory lesion and emphasized the potency of corticosteroid therapy which resulted into immediate, spectacular betterment of clinical presentation in 2 cases. Finally, Lawton Smith and David Taxdal in 1966 added five cases on top of the cases reported by Dr. Tolosa and Hunt et al. to initiate the use of the term THS as the painful ophthalmoplegia associated with $\mathrm{CN}$ paralysis that responded to corticosteroid treatment in 48 hours [4] [5] [6].

The cause of THS is still idiopathic, theories suggest THS be related to non-specific inflammation (non-granulomatous/granulomatous) in the region of the CS and/or SOF due to excess generation of fibroblasts and percolation of septa and wall of CS with lymphocytes and plasma cells, this can be ascertained to constant pain, which is the early exposition of this syndrome. Other potential causes could be aneurysms, traumatic insult, or neoplasms [2] [7] [8] [9].

TSH accounts for $23.2 \%$ among the frequent causes of CS syndrome [10]. Moreover, inflammatory conditions including THS, pachymeningitis and giant-cell arteritis are the major aetiology of acquired ocular motor nerve palsy by $21 \%$ [11]. Therefore, in the mounting of CS pathology, neuronal components within the CS, including the 3rd, 4th, and the divisions of the 5th CN (Ophthalmic and maxillary) are susceptible to damage [12].

The incidence of THS is approximately 1 to 2 cases per million [13]. There is no gender predilection among adults with THS and cuts across the ages from the 1 st to the 8th decades of life [13] [14]. Comparatively, in pediatric group THS seems to be exceedingly rare but follows a related course as adult group [15]. However, age of onset is 11 years by median though there has been a case report of 6 years old child reported, and predominantly affect female children than males, symptomatically most frequently presenting with 3rd CN involvement, followed by 6 th, 4 th, occasionally 5 th $\mathrm{CN}$, and rarely seventh $\mathrm{CN}$ involvement has been published in children, while $1 / 3$ the patients may report multiple $\mathrm{CN}$ 
palsy [16] [17] [18].

THS is essentially a pathology of exclusion therefore any patients presenting with one-sided PO without any other neurological anomaly should undergo a proper and extensive medical assessment to avoid several readmission due to misdiagnosis [9] [19] [20]. Lately, some authors rose a debate on the controversy of the term TSH attributable to its diagnostic challenges and dilemmas towards exclusion of other granulomatous presenting conditions considering that in actual clinical practice corticosteroid trial is initiated before biopsy under the umbrella of TSH impinging a risk of delayed diagnosis or misdiagnosis, therefore, proposing the retirement of the term or be reserved only for the syndromes that get resolved with treatment with no a precise diagnosis, alternatively, suggesting a term "painful ophthalmoplegia resolving with corticosteroid" to be used instead, in the same context clinicians, got reminded to repeatedly examine the diagnosis periodically [21] [22]. Furthermore, the controversy of whether to withdraw the term TSH or establishing clear diagnostic and management guidelines is the matter of future discussions.

Additionally, there is predominant importance of reportage of new cases and providing critical data concerning the course of the disease and the therapeutic outcomes for the rare diseases like THS, since contemporary digital angiography methods were inaccessible when THS was initially reported, and there are seldom reports regarding high-resolution angiographic data. Ultramodern tomography in particular magnetic resonance imaging (MRI) has simplified viewing the pathology of the orbital apex (OA) and CS and for ruling out other etiologies of PO, in the same way, facial pain overlapping syndromes including TSH is quite rare, and some neurologists or pain experts don't see such patients in their whole working career, therefore specialists should be familiarized with it since significant cases with likely fatal consequences have been published [3] [18] [23] [24]. In that context, this article is intended to provide the most recent understanding of diagnostic challenges facing THS in terms of presenting symptoms, differential diagnosis, laboratory investigations, imaging and therapeutic approaches.

\section{Diagnosis of THS}

\subsection{Presenting History/Symptoms}

Gathering THS manifesting symptoms chronologically, ease the diagnosis process of THS as summarized in Table 1. On the basis of the 2018 IHS diagnostic criteria, symptomatically THS presents with orbital/periorbital cephalgia which occur unilaterally and develop with paresis of the CNs III, IV, and VI or antedate by $\leq 2$ weeks, through case reports, extra involvement of the 5th $\mathrm{CN}$ Ophthalmic division, Facial nerve or Vestibulocochlear nerves have been reported, nevertheless, the pupil sympathetic branch at times is affected, the pain and paresis should eventually resolute with adequate corticosteroids therapy and shouldn't be outshined by other ICHD-3 diagnoses [1] [18] [25]. On the contrary 
Table 1. Diagnostic approach to exclude Tolosa-Hunt syndrome mimics.

\begin{tabular}{|c|c|c|}
\hline \multicolumn{3}{|c|}{ Diagnostic approach for excluding Tolosa hunt syndrome mimics } \\
\hline APPROACH & INQUIRE/LOOK FOR/PERFORM & POSSIBLE MIMICS TO BE EXCLUDED \\
\hline \multirow{7}{*}{$\begin{array}{l}\text { HISTORY } \\
\text { TAKING }\end{array}$} & Onset (Acute, Subactute) & $\begin{array}{l}\text { Pituitary Adenoma [26], Orbital apex syndrome [2] [28] [29], } \\
\text { IgG4-related disease [30] }\end{array}$ \\
\hline & Associated Symptoms & $\begin{array}{l}\text { Acute Orbital Myositis [31], Cryopyrin-associated } \\
\text { periodic fever syndrome [36] [37], }\end{array}$ \\
\hline & & Extranodal NK/T-cell lymphoma [38], Garcin syndrome [41] \\
\hline & Localization & Trochleodynia [33] \\
\hline & Progression & Carotid-cavernous fistula \\
\hline & Past drug history & Orbital Aspergillosis [44], THS in Pregnancy [46] \\
\hline & Past medical history & Burkitt lymphoma metastasis [28] [45] \\
\hline \multirow{4}{*}{$\begin{array}{l}\text { PHYSICAL } \\
\text { EXAMINTION }\end{array}$} & Diminished tendon reflex + extra-ocular movement & Miller Fisher Syndrome [53] \\
\hline & Visual acuity diminish, loss of nasal vision bilaterally & Clival chordoma [19] [56] \\
\hline & Fluorescein angiography & Eales' disease [57] \\
\hline & Slit-lamp examination & Sclerokeratitis, Takayasu's arteritis [58] \\
\hline \multirow{6}{*}{$\begin{array}{c}\text { LAB } \\
\text { AND } \\
\text { IMAGING }\end{array}$} & Voltage-gated calcium channel antibody & Lambert Eaton myasthenic syndrome \\
\hline & $\begin{array}{l}\text { Serum creatinine kinase }(\mathrm{CK}) \\
\text { and lactate dehydrogenase (LDH) }\end{array}$ & Orbital myositis \\
\hline & $\begin{array}{l}\text { Acetylcholinesterase receptor antibody } \\
\text { and repititive nerve stimulation }\end{array}$ & Myasthenia Gravis [43] \\
\hline & Antibodies against GQ1b and GT1a & Miller Fisher Syndrome [53] \\
\hline & Biopsy & Confirmation criteria [1] [17] [21] [28] [54] \\
\hline & MRI & Confirmation criteria [1] [28] \\
\hline
\end{tabular}

atypical bilateral presentation of THS, a pituitary adenoma mimicker case has been reported with headache that initially involved unilateral (the right orbit), followed by diplopia and retro-orbital pain on the very side, several days later identical symptoms experienced in the left eye, prompting the probability of the bilateral infiltration of the SOF and CS [26]. Morever, another atypical case of periorbital pain bilaterally in THS has been noted in 47 years man, who presented with periorbital pain associated with horizontal and vertical paralysis of field of vision with initial right side localization but 3 - 4 days later spread to the left side, alerting clinicians to consider TSH as the differential diagnosis for cases with bilateral PO [27].

Onset of TSH pain is usually acute experienced by a sufferer as boring, intense, sharp, piercing, severe, and shooting, besides, absence of pain can assist ruling out Orbital apex syndrome which may mimic THS by ophthalmoplegia, brow hypoaesthesia and the upper eyelid drop, in the same way, absence of pain and negative MRI for the ophthalmoplegic patients with recurrent CN III, IV and Ophthalmic division of $\mathrm{CN}$ V palsies can be helpful in precluding Polyneuritis Cranialis (PNC) with several similar features with THS [2] [28] [29]. Yet, 
subacute presentation implies slow development of orbital pseudotumor and lacrimal gland enlargement, this should direct physician towards IgG4-related disease [30].

Nonetheless, sudden onset of orbital pain which is worsened by ocular motion, conjunctiva redness, edematous lid and minimal proptosis can assist clinically distiction of THS from Acute Orbital Myositis [31]. Some patients may report deep seated pain retro-orbitally with down radiation to the neck [32]. Localized trochlea pain/tenderness as pointed by a patient which is aggravated by stress physically or emotionally or contraction of superior oblique (SO) muscle and headache on the front side from the superior nasal orbit extending periorbitally to ipsilateral boundaries is useful to distinguish clinically trochleodynia from THS, therefore in the deficit of a $\mathrm{CN}$ palsy neuroimaging is not recommend for trochleodynia exclusion [33].

Additionally, abnormal forehead tingling sensation (Paresthesia) may be noted implicating the involvement of the $\mathrm{CN} \mathrm{V}$ first (ophthalmic) division, this poses a diagnostic dilemma especially in diabetes mellitus (DM) as DM presence is not confirmatory for the diabetic ophthalmoplegia (DO) diagnosis, nonetheless due to their similarities in clinical sequel, unclear diagnostic criteria and etiologies, boundaries between DO, DM with THS, and DM with recurrent painful ophthalmoplegia neuropathy (RPON) remains challenging, of note for the clinician, concurrent onset of multiple $\mathrm{CN}$ palsy in DM is improbable diabetic microangiopathy warranting further evaluation and imaging of the ipsilateral CS and/or OA [9] [25] [34]. THS symptoms are characterized by on and off progression with monthly or years recurrences, resulting into readmissions, this may mimic initial presentation of Carotid-cavernous fistula (CCF), therefore, the attending doctor should consider digital subtraction angiography (DSA) to preclude possibility of posterior-draining CCF [20] [32] [35].

It is compulsatory to perform thoughtful evaluation of the associated systemic symptoms, as presence of fevers, hives rash, hearing loss, red eyes, joint pain following history of unilateral PO and diplopia is likely to be cryopyrin-associated periodic fever syndrome rather than THS hence confirmatory genetic testing for the (Q703K) mutation of the cryopyrin/NLRP3 gene and inflammatory markers should be considered [36] [37]. By the same token, pyrexia of unknown origin and nosebleed which may mistakenly thought to be a common cold, when followed with symptoms/signs of CS syndrome mimicking THS such as PO, diplopia, 3rd and 6th $\mathrm{CN}$ palsy unilaterally, is convincingly a metastases of a rare but fatal malignant lymphoma, the Extranodal NK/T-cell lymphoma which is prevalent in Mexico, Asia, South America, therefore unexceptionable evidence for the existence of abnormal, focal lesions should be sought from the PET-CT and MRI, as early treatment is associated with good prognosis [38].

Prompting for the associated features should follow after exhausting the presenting complains [39]. In the most recent retrospective case series regarding isolated sphenoid sinus inflammatory diseases involving 23 patients with new 
vision loss/diplopia and unremitting headache or painful orbit, it was observed that twenty out of the twenty three patients (87\%) presented with pain (headache and ocular pain) with oculomotor and abducens nerve palsies similar to THS presentation, but associated symptoms of one sided ocular disturbance in the form of blurred vision in $65 \%$ of the cases, two-sided binocular diplopia in $47 \%$, ptosis in $17 \%$ and proptosis in $4 \%$ of the cases with CT/MR confirmation for pathology was crucial to rule out THS [40]. Never the less associated history of rapid weight loss even without the history of smoking in a patient with headache, diplopia and $\mathrm{CN}$ palsies, should warn a physician for the possibilities of Guillain-Alajouanine-Garcin syndrome (Garcin syndrome) which is a metastatic tumor with bone destruction, therefore CT Scan should be ordered for confirmation of osteoblastic lesion in base of the skull [41].

Initiating high dose corticosteroid therapy setting of TB might be very dangerous, therefore, it is mandatory to preclude prior history of dry chronic cough in patients from tuberculosis (TB) endemic regions even without associated history of B symptoms, hemoptysis or pleurisy in patients with unilateral features of CS syndrome mimicking THS, by running rigorous investigations including histology for the possibilities of necrotizing granulomas, tissue microscopy and staining for acid-fast bacilli, MPT64 antigen and TB PCR for MTB complex, and Cerebrospinal fluid (CSF) TB PCR [42]. Occasionally, myasthenia gravis (MG) co-exist with THS and pose a confounding clinical picture and a challenging treatment approach, as MG and THS both present with ptosis and diplopia their coexistence creates diagnostics challenges, moreover, in the presence of unilateral facial numbness, retro-orbital pain, diplopia, confirmed unilateral granulation tissue in MRI Brain, weakness and fatigable extraocular muscles, postive Acetylcholinesterase receptor antibody and repititive nerve stimulation a combined diagnosis of THS and MG is inevitable [43].

Past drug/treament history should be considered when trying to close-out THS mimics, physicians should ask for the history of use of immunosuppressant drugs such as mycophenolate mofetil, excessive use of antibiotics, cortico-steroids, chemotherapy or history diabetes, as these may predispose to orbital aspergillosis [44]. Prompting for the past medical history on HIV status in THS is of great importance when ruling out other possibilities of PO, as Burkitt lymphoma metastatic to the CS may present with diplopia, ptosis, numbness in the CN 5 (ophthalmic) and occasionally without typical B symptoms of lymphoma, on-occasion this may associated with severe abdominal pain, therefore before initiation of cortcosteroids a positron emission tomography (PET-CT) is waranted for the evidence of fluorodeoxyglucose uptake in the intra-abdominal organs such as terminal ileum, moreover PO should be regarded as one of the early presentation of HIV-associated lymphoma and normaly with poor corticosteroid response and relapsing eye symptoms [28] [45].

Similarly, among the pregnant women THS presents with headache, nausea and diplopia unilaterally, the incidence has been linked to recent increased tendency of progesterone supplementation for the spontaneous abortion and pre- 
mature deliveries avoidance, the mechanism behind being the immune modulation outcomes of pregnancy moderated by progesterone and estrogen by expressing angiogenic factors in the inflamatory tissue and lower the apoptosis of granulomatous cells [46].

\subsection{Physical Examintion/Signs}

On neurological examination paresis of the ipsilateral oculomotor $\mathrm{CN}$ may be detected in the form of ptosis due to levator palpebrae superioris muscle denervation, inferior and lateral deviation of the eye due to unobstructed effect of the lateral rectus (LR) and superior oblique (SO) muscles, double vision(diplopia) because of ciliary muscles palsy especially when the gaze is towards the paretic muscle and pupil dilatation due to deprivation of the sphincter pupillae parasympathetic supply [31] [47] [48].

Correspondingly, THS is uncommon etiology of painful diplopia, lesion of the CN IV produces vertical diplopia in the ipsilateral eye intensified by downward gaze, ocular extorsion, downward gaze insufficiency, and head tilting compensatory for extorsion, therefore determining whether diplopia is monocular or binocular is critical in establishing the aetiology, as binocular diplopia is commonly resulted by extraocular muscle impairment while localized ocular pathology or refractive error frequently result in monocular diplopia, in people $>$ 50 years giant cell arteritis predominantly causes of new-onset headache or ocular disturbances, therefore prompt more for presence of fever, malaise, elevated inflammatory markers, and confirmatory temporal artery biopsy [49] [50].

In the same manner, unilateral abduction deficit and horizontal diplopia on gazing towards the lateral rectus with palsy is consistent with abducent nerve paralysis [51] [52]. A combination of diminished tendon reflex and extra-ocular movement disturbance advocate Miller Fisher Syndrome which mimics THS [53]. Persistent ipsilateral somatosensory loss/forehead paresthesia and decreased pinprick sensation owing to the subdivision of trigeminal nerve (ophthalmic) lesion may also be detected on examination [25] [54] [55].

Occasionally, visual acuity may be diminished because of periorbital pain hindering co-operation, while on visual field examination, loss of nasal vision bilaterally may be identified as in cases of clival chordoma a skull-based tumor which should be confirmed pathologically, along with its other features of prolonged duration and gradual radiographic furtherance [19] [56].

A detailed ophthalmological examination should not complemented without fluorescein angiography to exclude Eales' disease which is steroid responsive vasculopathy PO mimicking THS, specifically retinal or optic disc neovascularization, peripheral venous leakage, oedematous macular area, perivasculitis of the retina and non-perfused areas should be examined [57]. Slit-lamp examination for the inspection should be done to preclude the possibilities of sclerokeratitis bilaterally and optic atrophy as manifestation of Takayasu's arteritis (TA) which may mimick THS by 6th and 7th CNs palsies and corticosteroid responsiveness, hence confirmatory CT aortic angiography should be ordered [58]. 


\subsection{Imaging in THS}

IHS 2018 diagnostic criteria requires MRI demonstration of CS or SOF/OA granulomatous inflammation (GI) ipsilateral to the presenting headache as confirmatory diagnostic technique for THS, as it can distinguish THS from its mimics, such as vascular disorders, tumors, plasma cell granuloma, ophthalmoplegic migraine, idiopathic hypertrophic cranial pachymeningitis, tuberculoma, sarcoidosis, other GIs and OA disorders such as OA syndrome, SOF syndrome and CS syndrome [1] [28] [59] [60].

MRI findings of THS may reveal thickening changes of the CS due to the soft tissue abnormality with or without entanglement of the internal carotid(IC) artery, $\mathrm{SOF}, \mathrm{OA}$, or optic $\mathrm{CN}$, the granulomatous lesion appears as $\mathrm{T} 1$ isointensity sequence and iso hypointensity apperance in T2 sequences with diffusion abnormality and shows significant contrast involvement following contrast injection of the medium intravenously, Figure 1 [2] [19] [56] [61] [62] [63]. Tolosa Hunt MRI protocol (contrast-enhanced MRI) demonstrate with high resolution lesion details of the soft tissue and therefore should be used in all the THS suspected cases as the cornerstone of diagnosis because the conventional MRI seems to offer an inadequate proof of the GI, On the contrast, case series studies has indicated that about $50 \%$ of all THS cases have a negative MRI, hence, proposed that GI may be present even if MRI is negative. [7] [8] [20] [64]. Recent researches have revealed that restricted diffusion can delineate the extent of THS as precisely as the current cornerstone modality of choice (the Gadolinium-enhanced MRI) in portraying the degree of the lesion and guide treatment response by virtue of intrusion by inflammatory cells makes the inflammatory regions more evident on DWI, hence further studies of these lesions on DWI can pave a way into significant reduction total scan duration, on the side, Constructive interference in steady-state (CISS) MRI has recently been proved to have high sensitivity as well for diagnosing and follow up THS [55] [65].

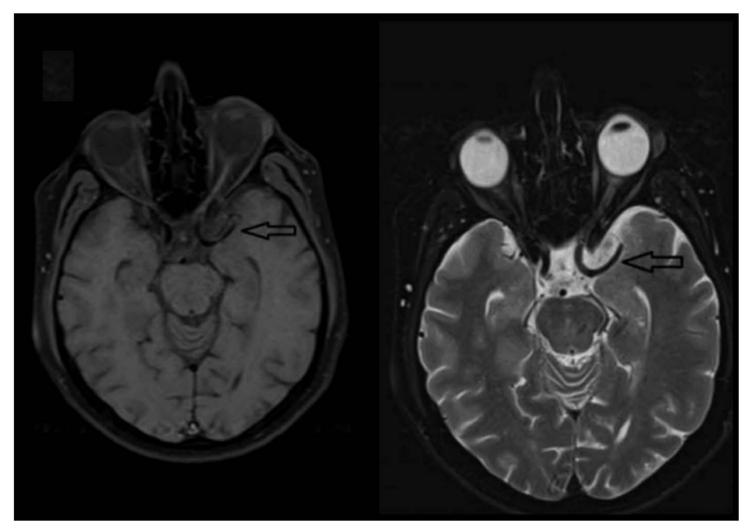

(a)

(b)

Figure 1. MRI results of a 59-year-old male, who presented with unilateral left sided periorbital cephalgia, diplopia and ptosis. Diagnosed with left sided THS. (a) Axial T1-weighted; (b) Axial T2-weighted, post-contrast sequence displaying soft tissue enhancement (black arrows) [67]. 
Nevertheless, reliably of MRI technology on identification of THS by demonstration of GI is still challenging as number of false-negative/positive diagnoses are identified, some cases which were initially diagnosed with THS and confirmed with MRI happened to be reassessed later and assigned to other disorders, therefore it is critically suggested that patient follow-up time should be lengthened to 2 years in patients with benign MRIs, Moreover MRI or biopsy should not be mandatory criteria for diagnosis as opposed to the current IHS 2018 guidelines [51] [66].

Angiography is ventured primarily to eliminate the possibility of CCF or CS segment aneurysms, moreover cerebral angiographic findings have been beneficial in diagnosing focal narrowing of the IC artery, DSA may reveal draining CCF,CT aortic angiography may reveal peculiarities of aortoarteritis to preclude TA, likewise PO chiefly of the CN III, guarantee vascular imaging owing to an underlying aneurysm as infrequently an aneurysm may arise in combination with inflammatory transformation of the CS and ICA [3] [16] [35] [58] [67].

\subsection{Laboratory Studies in THS}

THS prevails as a pathology of exclusion Figure 2, consequently when it's suspected on grounds of clinical presentation and MRI, then blood, biopsy and cerebrospinal fluid (CSF) investigations should be though for ruling out PO etiology, further, infectious events can be precluded by white blood counts, C-reactive protein and erythrocyte sedimentation rate, while, CSF examination can be required to asses opening pressure, cell count and differential, fungal, glucose and/or bacterial cultures, gram stain, cytology, CSF angiotensin-converting enzyme (ACE) levels for precluding neurosarcoidosis, systemic lupus erythematosus, vasculitides and Wegener's granulomatosis, furthermore, voltage-gated calcium channel antibody for screening Lambert Eaton myasthenic syndrome, serum creatinine kinase $(\mathrm{CK})$ and lactate dehydrogenase (LDH) for orbital myositis, antibodies against GQ1b and GT1a for the differentiation of initial, painless THS and MFS, other important tests include, Lyme titre screen, mycoplasma antibodies, HIV antigen, and West Nile polymerase chain reaction, glucose, hemoglobin A1C, thyroid hormones, renal and liver function tests [3] [20] [43] [53] [66] [68]. Precluding Merkel cell carcinoma (MCC) warrants screening for immunuohistochemical slides for tumor CD56, cytokeratin CAM 5.2 and cytokeratin 20, TTF-1, antigen (LCA), S-100, and melan-A [69].

Validation of GI of the CS, SOF or orbit by biopsy or MRI is currently regarded by the IHS as mandatory criteria for confirmation of THS diagnosis, CS biopsy is an advanced and complex procedure that requires to be conducted by a skilled neurosurgeon, if performed in conjunction with adjunctive utilization of visual guidance routines, insult to vital organs such as CN II and ophthalmic artery can be arrested, although it is beneficial when diagnosis is uncertain for precluding conditions such as actinomycosis by the existence of sulfur granules histologically, frequently in practice, biopsy is seldom done ere a trial 


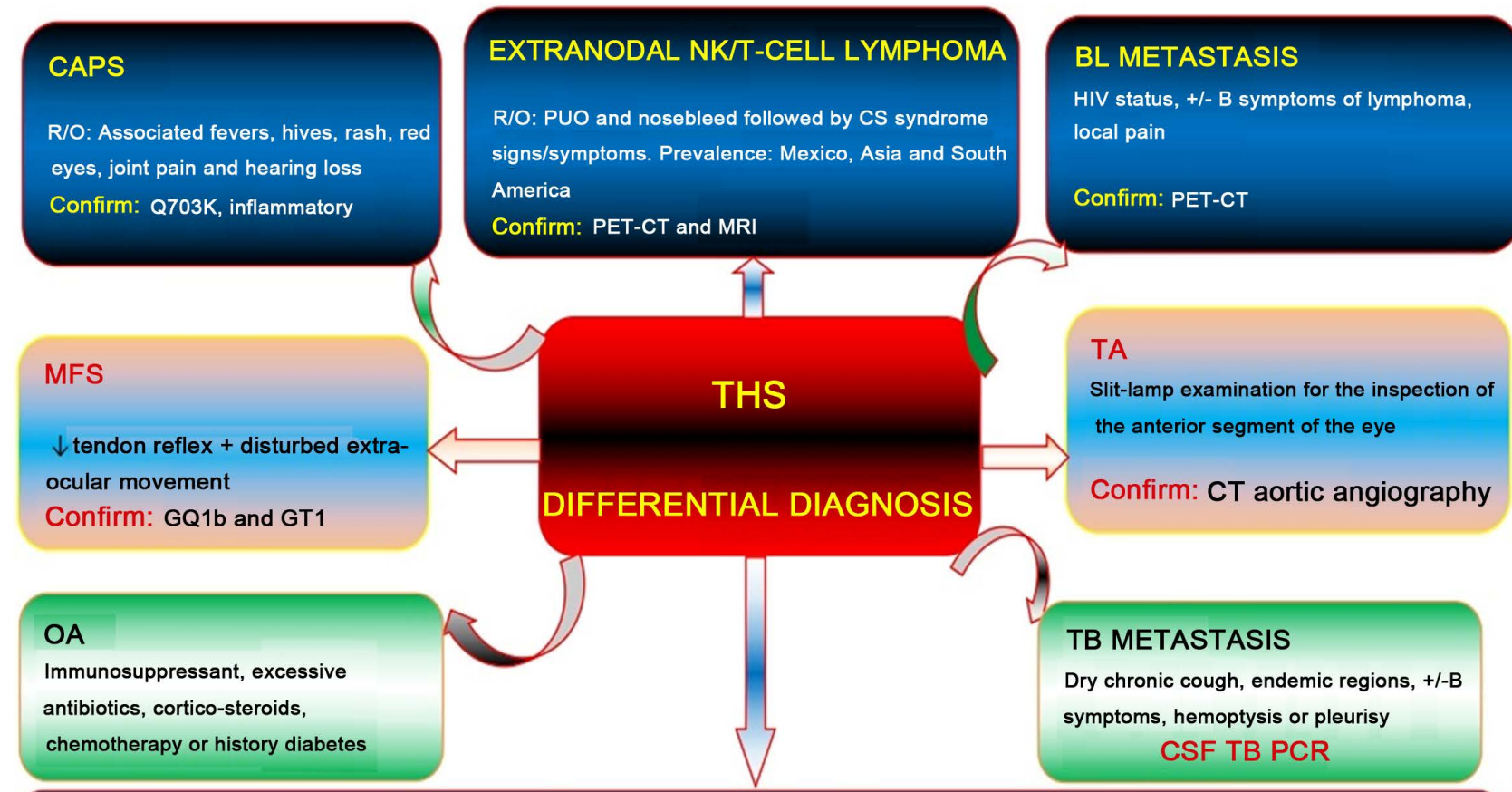

OTHERS: CCF, Garcin syndrome, myasthenia gravis, giant cell arteritis, clival chordoma, neurosarcoidosis, actinomycosis, Carotid-cavernous thrombosis, DM, Eales' disease, Orbital apex syndrome, pituitary adenoma, Wegener's granulomatosis, pseudotumor, Intracavernous carotid artery aneurysm, isolated sphenoid sinus inflammatory diseases, Merkel cell carcinoma, recurrent painful ophthalmoplegia neuropathy

ABBREVIATION: Tolosa-Hunt syndrome (THS), cryopyrin-associated periodic fever syndrome (CAPS), pyrexia of unknown origin (PUO), positron emission tomography (PET-CT), Miller Fisher Syndrome (MFS), Carotid-cavernous fistula (CCF), Takayasu's arteritis (TA), orbital aspergillosis, BL-burkitt Lymphoma, Diabetes Melitus (DM)

Figure 2. Differential diagnosis of Tolosa hunt syndrome. Summarizing 26 differential diagnosis for Tolosa Hunt Syndrome, seven of them (first three rows) summarized based on distinction details of presenting history, physical examination and investigation, the last row shows the abbreviations of the terms.

of corticosteroids owing to location, smaller lesions and a concern for the likelihood of damaging the enclosing stuructures [1] [17] [21] [28] [54] [59] [66] [70].

\section{Management of THS}

Generally corticosteroid therapy such as prednisolone (PSL), methylprednisolone (mPSL) and dexamethasone, given either orally or intravenously for THS results in climactic improvement in pain which is usually apparent in a few days, notwithstanding the fact that corticosteroids are the cornerstone of treatment for THS, there are controversies concerning effective course, route of administration, optimal dosage, period of therapy and treatment in special groups such as pregnant women and children, a case with fastest recovery has been portrayed in a young male with retro obital pain and ocular motilities resolving in 12 hours of the first high dose IV mPSL of $500 \mathrm{mg}$ BID for 3 days and nearly absolute normalization in 4 days and kept on 7 days daily maintenance dose of prednisone 60 $\mathrm{mg}$, on the contrary another case on IV mPSL of $1000 \mathrm{mg}$ for 3 days reported 
instant lessening of pain but diplopia persisted and eventually PO recurred, In children mPSL of $1 \mathrm{mg} / \mathrm{kg} / \mathrm{d}$ for 7 days and gradual tapering up to three months and the alternative same dose for 14 days tapered slowly over 1 month have been proved to be useful in different occasions with no relapses remarked for more than 3 years of clinical and MRI follow-up, of note, more researches are obligated to ascertain the optimal corticosteroid course for the therapy in adults and pediatric population with THS [3] [7] [18] [20] [27] [71].

Infusion with $300 \mathrm{mg}$ of a tumor necrosis factor-alpha antibody (infliximab) has been reported to result into dramatic and tenacious recovery in patients who previously reported recurrence of headache and diplopia on tapering prednisone to beneath $50 \mathrm{mg}$ and cushingoid hallmarks, edematous legs, acne and hyperglycemia on maintanance $50 \mathrm{mg}$ daily, hence, controlled researches should be conducted to systematize the usage of infliximab in THS, other alternative therapies include azathioprine and methotrexate which irregularly demands to be supplemented to sustain the primary response to glucocorticoids. On the other hand, numerous systemic side effects of steroids, such as gastrointestinal problems, moon face, and generalized edema, sinusitis and pituitary abscess have been noted moreover steroid psychosis may arise in severe cases, the demonstration of steroid psychosis following corticosteroid treatment is dose-dependent such as in mPSL of $1000 \mathrm{mg} /$ day for 3 days, consequently, corticosteroid treatment for aged patients necessitates attention and dose modulation considering likehood of negative drug effects, if corticosteroids are contraindicated or serious side effects are present, corticosteroid treatment has to be suspended [7] [9] [15] [54] [72] [73].

Nonetheless, acupuncture has been widely utilized for management of ophthalmological conditions particularly ophthalmoplegia in China, Multiple clinical trials show that acupuncture may improve the recovery of extra-ocular muscles in ophthalmoplegia cases farther researches are warranted to appraise the effectiveness and safety of acupuncture for THS ophthalmoplegia [74].

Microsurgery can be utilized for benign lesion evacuation inside the CS in cases with unmanageable headaches, nonresponsiveness to steroid and analgesic with relative safety, though there is no straight forward indication for microsurgical removal of such lesions, decision should come after weighing all perspectives of the victim and lesion nonetheless, post-surgically there is reported feeling of caressing downward of warm water beneath the skin on the unilateral forehead and permanent CN VI palsy. On the other hand, radiation therapy for medically unmanageable recurrences of THS has been employed recently, Gamma Knife radiosurgery (GKRS) which is a single-fraction radiation therapy offers a very dramatic improvement by applying very low-dose radiation volume into the normal tissues surrounding the target, can be utilized as an alternative treatment option for THS in cases where there is contraindication or intolerability of corticosteroid, although GKRS bears the hazard of late malignancy, the precisity of sub millimetre of GKRS, offers a very dramatic reduction of the 
low-dose radiation impact into the normal surrounding tissues therefore the risk of secondary on cogenesis is extensively diminished [59] [75] [76].

\section{Conclusions}

THS is an unusual diagnosis of exclusion which necessitates consultations from several specializations. As THS diagnosis encounters hurdles and dilemmas multi-specialization consultations from the emergency physicians, neurologists, neurosurgeons, neuro-hospitalists, ophthalmologists and otolaryngologists are necessitated in overcoming the challenges.

Moreover, obtaining details of THS presenting symptoms chronologically, physical examination maneuvers, laboratory investigations, and imaging techniques help to subdue diagnostic expostulations and quandaries facing THS as summarized in Table 1.

Application of ultramodern tomography techniques particularly Tolosa Hunt MRI protocol (contrast-enhanced MRI), restricted diffusion and CISS MRI has lately proved to be precise investigations for THS diagnosis and follow up hence assists in overcoming diagnostic barriers by simplifying viewing the pathology of the orbital apex (OA) and CS and other etiologies of PO and facial pain overlapping syndromes.

Furthermore, validation of GI of the CS, SOF or orbit by biopsy or MRI is currently regarded by the IHS as mandatory criteria for confirmation of THS diagnosis, CS biopsy is an advanced and complex procedure when conducted by skilled neurosurgeon that yields a better result with fewer complications.

Corticosteroids persevere as a cornerstone therapy for THS through standardized guidelines are needed, however, infliximab (300 mg infusion), azathioprine, methotrexate, and acupuncture exist as alternative therapies, lately surgical techniques have been employed although permanent CN VI palsy and risk of late malignancy have been stated.

\section{Conflicts of Interest}

The authors declare no conflicts of interest with reference to the publishing of this review article.

\section{References}

[1] Vincent, M. and Wang, S. (2018) Headache Classification Committee of the International Headache Society (IHS) the International Classification of Headache Disorders, 3rd Edition. Cephalalgia: An International Journal of Headache, 38, 1-211. https://doi.org/10.1177/0333102417738202

[2] Amrutkar, C. and Burton, E.V. (2019) Tolosa-Hunt Syndrome. StatPearls. StatPearls Publishing StatPearls Publishing, Treasure Island.

[3] Ravindran, K., Schmalz, P., Torun, N., Ronthal, M., Chang, Y.M. and Thomas, A.J. (2018) Angiographic Findings in the Tolosa-Hunt Syndrome and Resolution after Corticosteroid Treatment. Neuro-Ophthalmology, 42, 159-163. https://doi.org/10.1080/01658107.2017.1365268 
[4] Tolosa, E. (1954) Periarteritic Lesions of the Carotid Siphon with the Clinical Features of a Carotid Infraclinoidal Aneurysm. Journal of Neurology, Neurosurgery, and Psychiatry, 17, 300-302. https://doi.org/10.1136/jnnp.17.4.300

[5] Hunt, W.E., Meagher, J.N., Lefever, H.E. and Zeman, W. (1961) Painful Opthalmoplegia. Its Relation to Indolent Inflammation of the Carvernous Sinus. Neurology, 11, 56-62. https://doi.org/10.1212/WNL.11.1.56

[6] Smith, J.L. and Taxdal, D.S. (1966) Painful Ophthalmoplegia. The Tolosa-Hunt Syndrome. American Journal of Ophthalmology, 61, 1466-1472. https://doi.org/10.1016/0002-9394(66)90487-9

[7] Penić, S.Z., Lisak, M., Gregurić, T., Hećimović, H. and Kes, V.B. (2017) Tolosa-Hunt Syndrome: Case Report. Acta Clinica Croatica, 56, 331-337. https://doi.org/10.20471/acc.2017.56.02.18

[8] Akpinar, Ç.K., Özbenli, T., Doğru, H. and İncesu, L. (2017) Tolosa-Hunt Syndrome: Cranial Neuroimaging Findings. Noropsikiyatri Arsivi, 54, 251-254. https://doi.org/10.5152/npa.2016.13791

[9] Chakraborty, P.P., Patra, S., Barman, H. and Biswas, S.N. (2017) Cranial Neuropathies in Uncontrolled Diabetes: May Not Always Be Due to Diabetic Microangiopathy. BMJ Case Reports, 2017, bcr2017220054.

https://doi.org/10.1136/bcr-2017-220054

[10] Bhatkar, S., Goyal, M.K., Takkar, A., Mukherjee, K.K., Singh, P., Singh, R. and Lal, V. (2017) Cavernous Sinus Syndrome: A Prospective Study of 73 Cases at a Tertiary Care Centre in Northern India. Clinical Neurology and Neurosurgery, 155, 63-69. https://doi.org/10.1016/j.clineuro.2017.02.017

[11] Choi, K.D., Choi, S.Y., Kim, J.S., Choi, J.H., Yang, T.H., Oh, S.Y., Choi, J.Y., et al. (2019) Acquired Ocular Motor Nerve Palsy in Neurology Clinics: A Prospective Multicenter Study. Journal of Clinical Neurology (Korea), 15, 221-227. https://doi.org/10.3988/jcn.2019.15.2.221

[12] Durgam, A., Hussain, H., Nakatsuka, A.S. and Raghuram, K. (2019) Isolated Trochlear Nerve (Cranial Nerve IV) Palsy in a Patient with Carotid-Cavernous Fistula. BJR Case Reports, 5, Article ID: 20180028. https://doi.org/10.1259/bjrcr.20180028

[13] Iaconetta, G., Stella, L., Esposito, M. and Cappabianca, P. (2005) Tolosa-Hunt Syndrome Extending in the Cerebello-Pontine Angle. Cephalalgia, 25, 746-750. https://doi.org/10.1111/j.1468-2982.2005.00924.x

[14] Kline, L.B. and Hoyt, W.F. (2001) The Tolosa-Hunt Syndrome. Journal of Neurology, Neurosurgery, and Psychiatry, 71, 577-582. https://doi.org/10.1136/jnnp.71.5.577

[15] Rodriguez-Homs, L.G., Goerlitz-Jessen, M. and Das, S.U. (2019) A 17-Year-Old Girl with Unilateral Headache and Double Vision. Journal of Investigative Medicine High Impact Case Reports, 7, 4-6. https://doi.org/10.1177/2324709619838309

[16] Pérez, C.A. and Evangelista, M. (2016) Evaluation and Management of Tolosa-Hunt Syndrome in Children: A Clinical Update. Pediatric Neurology, 62, 18-26. https://doi.org/10.1016/j.pediatrneurol.2016.06.017

[17] Douleh, D.G., Morone, P.J., Johnson, J.E., Paueksakon, P. and Wellons, J.C. (2016) Actinomycosis Mimicking Tolosa-Hunt Syndrome in a 6-Year-Old Boy: Case Report. Pediatric Neurosurgery, 51, 214-217. https://doi.org/10.1159/000444940

[18] Tsirigotaki, M., Ntoulios, G., Lioumpas, M., Voutoufianakis, S. and Vorgia, P. (2019) Tolosa-Hunt Syndrome: Clinical Manifestations in Children. Pediatric Neurology, 99, 60-63. https://doi.org/10.1016/j.pediatrneurol.2019.02.013 
[19] Prete, B. and Sowka, J. (2017) Painful Ophthalmoplegia as an Initial Presentation of Sarcoidosis. Clinical and Experimental Optometry, 100, 291-293. https://doi.org/10.1111/cxo.12468

[20] Jarholm, J.A., Faiz, K.W., Nysted, T., Zarnovicky, S. and Kristoffersen, E.S. (2018) Orbital Pain, Ophthalmoplegia, and Oligoclonal Bands in the Cerebrospinal Fluid: A Case Report of Tolosa-Hunt Syndrome. Headache, 58, 758-760. https://doi.org/10.1111/head.13283

[21] Lubomski, M., Dalgliesh, J., Lee, K., Damodaran, O., McKew, G. and Reddel, S. (2018) Actinomyces Cavernous Sinus Infection: A Case and Systematic Literature Review. Practical Neurology, 18, 373-377. https://doi.org/10.1136/practneurol-2017-001844

[22] Lueck, C.J. (2018) Time to Retire the Tolosa-Hunt Syndrome? Practical Neurology, 18, 350-351. https://doi.org/10.1136/practneurol-2018-001951

[23] Evers, S. (2017) Facial Pain: Overlapping Syndromes. Cephalalgia, 37, 705-713. https://doi.org/10.1177/0333102417703761

[24] Okawa, S., Hanazono, A., Sugawara, M., Takahashi, S., Otani, T., Hanyu, N., et al. (2012) Contrast-Enhanced 3D FIESTA Imaging in Tolosa-Hunt Syndrome. Headache, 52, 822-824. https://doi.org/10.1111/j.1526-4610.2012.02089.x

[25] Lasam, G. and Kapur, S. (2016) A Rare Case of Tolosa-Hunt-Like Syndrome in a Poorly Controlled Diabetes Mellitus. Case Reports in Medicine, 2016, 4-7. https://doi.org/10.1155/2016/9763621

[26] Świątkowska-Stodulska, R., Stodulski, D., Babińska, A., Piskunowicz, M. and Sworczak, K. (2017) Bilateral Tolosa-Hunt Syndrome Mimicking Pituitary Adenoma. Endocrine, 58, 582-586. https://doi.org/10.1007/s12020-017-1422-2

[27] Kastirr, I., Kamusella, P. and Andresen, R. (2016) Bilateral Painful Ophthalmoplegia: A Case of Assumed Tolosa-Hunt Syndrome. Journal of Clinical and Diagnostic Research, 10, 16-17. https://doi.org/10.7860/JCDR/2016/16703.7496

[28] Baig, I., Webb, A., Crowell, E.L., Supsupin, E.P. and Adesina, O.O. (2019) Burkitt Lymphoma Masquerading as Tolosa-Hunt Syndrome in an HIV-Seropositive Patient. European Journal of Ophthalmology. https://doi.org/10.1177/1120672119842998

[29] Torres, A.R., Salvador, C., Mora, M., Mirchandani, S. and Chavez, W. (2019) Idiopathic Recurrent Polyneuritis Cranialis: A Rare Entity. Cureus, 11, e4488. https://doi.org/10.7759/cureus.4488

[30] Xiong, M. and Moy, W.L. (2018) Orbital Apex Syndrome Resulting from Mixed Bacterial Sphenoid Sinusitis. European Journal of Case Reports in Internal Medicine, 5, Article ID: 000905. https://doi.org/10.12890/2018 000905

[31] Ropper, A.H., Samuels, M.A., Klein, J.P. and Prasad, S. (2019) Adams and Victor's Principles of Neurology. McGraw-Hill Medical Pub. Division, New York.

[32] Murtaza, G., Konowitz, N., Lu, H., Faqah, A. and Kuruvilla, A. (2017) An Interesting Case of Tolosa-Hunt Syndrome in a Young Male. Journal of Investigative Medicine High Impact Case Reports, 5, 3-5. https://doi.org/10.1177/2324709616689478

[33] Tran, T.M., McClelland, C.M. and Lee, M.S. (2019) Diagnosis and Management of Trochleodynia, Trochleitis, and Trochlear Headache. Frontiers in Neurology, 10, 361. https://doi.org/10.3389/fneur.2019.00361

[34] Hamada, K., Sakurai, Y. and Sugimoto, I. (2016) Recurrent Painful Ophthalmoplegia in a Patient with Diabetes Mellitus: Is Ophthalmoplegia Associated with Di- 
abetes Mellitus? Cephalalgia, 36, 1397-1398. https://doi.org/10.1177/0333102416629240

[35] Li, S., Feng, B., Feng, Y., Pang, Z. and Lin, Y. (2019) Carotid-Cavernous Fistula (CCF) Presenting as Paroxysmal Painful Ophthalmoplegia. BMC Ophthalmology, 19, 2-5. https://doi.org/10.1186/s12886-019-1039-8

[36] Keddie, S., Parker, T., Lachmann, H.J. and Ginsberg, L. (2018) Cryopyrin-Associated Periodic Fever Syndrome and the Nervous System. Current Treatment Options in Neurology, 20, 43. https://doi.org/10.1007/s11940-018-0526-1

[37] Höhne, C., Schuh, E., Kümpfel, T. and Straube, A. (2016) Cryopyrin-Associated Periodic Fever Syndrome Manifesting as Tolosa-Hunt Syndrome. Cephalalgia, 36, 1392-1396. https://doi.org/10.1177/0333102416629239

[38] Mai, H.-C., Chen, D.-X., Lu, D. and Zhang, Y.-S. (2017) Extranodal Natural Killer/T-Cell Lymphoma Presenting as Cavernous Sinus Syndrome. Molecular and Clinical Oncology, 6, 543-546. https://doi.org/10.3892/mco.2017.1190

[39] Alastair Innes, J., Dover, A.R. and Fairhurst, Kn. (2018) General Aspects of History Taking. In: Macleod's Clinical Examination, 14th Edition, Elsevier Inc., Amsterdam, 9-17.

[40] Chen, L., Jiang, L., Yang, B. and Subramanian, P.S. (2017) Clinical Features of Visual Disturbances Secondary to Isolated Sphenoid Sinus Inflammatory Diseases. BMC Ophthalmology, 17, 237. https://doi.org/10.1186/s12886-017-0634-9

[41] Fukai, S., Okabe, N., Mine, H., Takagi, H. and Suzuki, H. (2018) Garcin Syndrome Caused by Sphenoid Bone Metastasis of Lung Cancer: A Case Study. World Journal of Surgical Oncology, 16, 46. https://doi.org/10.1186/s12957-018-1351-4

[42] Chih-Hui Tien, M., Ramachandran, P., White, O.B. and Lee, A.G. (2017) Not All Headaches Are Migraines. Survey of Ophthalmology, 62, 378-382.

https://doi.org/10.1016/j.survophthal.2016.05.001

[43] Majumdar, J., Mukhopadhyay, S., Sharan, A., Sengupta, S. and Ghosh, B. (2017) Tolosa-Hunt Syndrome and Ocular Myasthenia: A Rare Coexistence or Real Association. Journal of Association of Physicians of India, 65, 82-84.

[44] Liesse, A. and Salerno, D. (2018) Painful Ophthalmoplegia: Aspergillosis, Tolosa-Hunt and Other Causes. Journal of the Belgian Society of Radiology, 102, 1-2. https://doi.org/10.5334/jbsr.1496

[45] Takeuchi, S., Hagiwara, S., Nawashiro, H. and Shima, K. (2014) HIV-Associated Lymphoma Presenting with Painful Ophthalmoplegia. Asian Journal of Neurosurgery, 12, 341. https://doi.org/10.4103/1793-5482.145108

[46] Litwin, C.E. and Leung, A.S.O. (2017) Tolosa-Hunt Syndrome Presenting during Pregnancy Following Progesterone Administration. International Journal of Gynecology and Obstetrics, 137, 340-341. https://doi.org/10.1002/ijgo.12145

[47] Kose, A., Bozkurt, S., Umay, S.T., Karakulak, S. and Yilmaz, A. (2018) A Case of Headache, Double Vision and Ptosis in Emergency Department: Tolosa-Hunt Syndrome. Turkish Journal of Emergency Medicine, 18, 134-136. https://doi.org/10.1016/j.tjem.2017.10.004

[48] Lumley, J.S.P., Craven, J.L., Abrahams, P.H. and Tunstall, R.G. (2018) Bailey \& Love's Essential Clinical Anatomy. CRC Press, Boca Raton. https://doi.org/10.1201/9781315100722

[49] Nham, B. (2019) Hunting for a Cause of Painful Diplopia. Medical Journal of Australia, 210, 237-237.e1. https://doi.org/10.5694/mja2.50042

[50] Brueckner, J.K. and Fix, J.D. (2016) High-Yield Neuroanatomy. Wolters Kluwer, 
Philadelphia.

[51] Le Borgne, P. and Brunhuber, C. (2018) Woman with Headache and Retro-Orbital Pain. European Journal of Internal Medicine, 57, e6-e7. https://doi.org/10.1016/j.ejim.2018.05.006

[52] Champney, T. (2016) Essential Clinical Neuroanatomy. Wiley \& Sons, West Sussex.

[53] Oomura, M., Uchida, Y., Sakurai, K., Toyoda, T., Okita, K. and Matsukawa, N. (2018) Miller Fisher Syndrome Mimicking Tolosa-Hunt Syndrome. Internal Medicine, 57, 2735-2738. https://doi.org/10.2169/internalmedicine.0604-17

[54] Halabi, T. and Sawaya, R. (2018) Successful Treatment of Tolosa-Hunt Syndrome after a Single Infusion of Infliximab. Journal of Clinical Neurology (Korea), 14, 126-227. https://doi.org/10.3988/jcn.2018.14.1.126

[55] Wani, N.A., Jehangir, M. and Lone, P.A. (2017) Tolosa-Hunt Syndrome Demonstrated by Constructive Interference Steady State Magnetic Resonance Imaging. Journal of Ophthalmic and Vision Research, 12, 106-109. https://doi.org/10.4103/2008-322X.200171

[56] Ruff, M.W., Carabenciov, I.D., Johnson, D.R., Pollock, B.E., Parisi, J.E. and Klaas, J.P. (2018) A Cavernous Sinus Lesion Clinically Responsive to Steroids. Journal of Clinical Neuroscience, 53, 239-240. https://doi.org/10.1016/j.jocn.2018.04.020

[57] Mendonça, M.D., Guedes, M., Matias, G., Costa, J. and Viana-Baptista, M. (2017) Steroid-Responsive Painful Ophthalmoplegia: Tolosa-Hunt Syndrome, Eales Disease, or Both? Cephalalgia, 37, 191-194. https://doi.org/10.1177/0333102416631282

[58] Babu, K. and Parameswarappa, D.C. (2017) Atypical Tolosa Hunt Syndrome with Bilateral Sclerokeratitis and Optic Atrophy in Takayasu's Arteritis. American Journal of Ophthalmology Case Reports, 5, 41-43. https://doi.org/10.1016/j.ajoc.2016.11.006

[59] Chowdhury, F. and Haque, M. (2017) Microsurgical Management of Benign Lesions Interior to the Cavernous Sinus: A Case Series. Asian Journal of Neurosurgery, 12, 398. https://doi.org/10.4103/1793-5482.180892

[60] Goyal, P., Lee, S., Gupta, N., Kumar, Y., Mangla, M., Hooda, K., Mangla, R., et al. (2018) Orbital Apex Disorders: Imaging Findings and Management. Neuroradiology Journal, 31, 104-125. https://doi.org/10.1177/1971400917740361

[61] Yuliati, A. and Rajamani, K. (2018) Tolosa-Hunt Syndrome. The Neurohospitalist, 8, 104-105. https://doi.org/10.1177/1941874417714147

[62] Go, J.L. and Rajamohan, A.G. (2017) Imaging of the Sella and Parasellar Region. Radiologic Clinics of North America, 55, 83-101. https://doi.org/10.1016/j.rcl.2016.09.002

[63] Ferreira, T.A., Saraiva, P., Genders, S.W., Buchem, M.V., Luyten, G.P.M. and Beenakker, J.W. (2018) CT and MR Imaging of Orbital Inflammation. Neuroradiology, 60, 1253-1266. https://doi.org/10.1007/s00234-018-2103-4

[64] Gokharman, D. and Aydin, S. (2018) Magnetic Resonance Imaging in Orbital Pathologies: A Pictorial Review. Journal of the Belgian Society of Radiology, 101, 5. https://doi.org/10.5334/jbr-btr.1308

[65] Benjamin, P., Khan, F. and Mackinnon, A.D. (2017) The Use of Diffusion Weighted Imaging to Evaluate Pathology outside the Brain Parenchyma in Neuroimaging Studies. British Journal of Radiology, 90, Article ID: 20160821. https://doi.org/10.1259/bjr.20160821

[66] Mullen, E., Green, M., Hersh, E., Iloreta, A.-M., Bederson, J. and Shrivastava, R. 
(2018) Tolosa-Hunt Syndrome: Appraising the ICHD-3 Beta Diagnostic Criteria. Cephalalgia, 38, 1696-1700. https://doi.org/10.1177/0333102417745271

[67] Ceylan, M.E., Evrensel, A., Noyan, C.O. and Dönmez, A. (2016) Tolosa-Hunt Syndrome and Comorbidity of Obsessive Compulsive Disorder and Aortic Aneurysm. BMJ Case Reports, 2016, bcr2016216727. https://doi.org/10.1136/bcr-2016-216727

[68] Aquilina, N., Bugeja, V. and Zahra, C. (2018) A Case of Orbital Myositis Presenting With Dizziness. Open Access Macedonian Journal of Medical Sciences, 6, 1278. https://doi.org/10.3889/oamjms.2018.192

[69] Ho, K., Drew, P.A. and Chuquilin, M. (2017) Merkel Cell Carcinoma with Distant Metastasis to the Clivus Causing Symptoms Mimicking Tolosa-Hunt Syndrome: A Case Report and Literature Review. Frontiers in Neurology, 8, 409. https://doi.org/10.3389/fneur.2017.00409

[70] Unadkat, S., Rennie, C. and Grant, W. (2019) Endoscopic Transnasal Image-Guided Approach to Diagnosis in Orbital Apex and Optic Canal Lesions. The Journal of Laryngology \& Otology, 133, 501-507. https://doi.org/10.1017/S0022215119000975

[71] Flint Beal, M. and Hauser, S.L. (2018) Trigeminal Neuralgia, Bell's Palsy, and Other Cranial Nerve Disorders. In: Harrison's Principles of Internal Medicine, 20th Edition, McGraw-Hill, New York, 3166-3172.

[72] Ishikawa, R., Ishikawa, Y., Kubota, T. and Shirahama, K. (2018) A Case of Delirium in Tolosa-Hunt Syndrome during Corticosteroid Therapy. JA Clinical Reports, 4, 58. https://doi.org/10.1186/s40981-018-0193-y

[73] Takasuna, H., Sasaki, R., Shiraishi, M., Doi, M., Wakui, D., Ito, H., Tanaka, Y., et al. (2016) Steroid-Resistant Tolosa-Hunt Syndrome with a de Novo Intracavernous Aneurysm: A Case Report. Surgical Neurology International, 7, S779-S784. https://doi.org/10.4103/2152-7806.193925

[74] Ji, M., Qin, Y., Zi, Y., Wang, R., Meng, H., Yang, Z., Jin, M., et al. (2018) Acupuncture for Ophthalmoplegia: Protocol for a Systematic Review. Medicine, 97, e11065. https://doi.org/10.1097/MD.0000000000011065

[75] Lee, J.M., Park, J.S. and Koh, E.J. (2016) Gamma Knife Radiosurgery in Steroid-Intolerant Tolosa-Hunt Syndrome: Case Report. Acta Neurochirurgica, 158, 143-145. https://doi.org/10.1007/s00701-015-2648-5

[76] Kemeny, A.A. (2016) Tolosa-Hunt Syndrome: Another Legitimate Target for Radiosurgery? Acta Neurochirurgica, 158, 141.

https://doi.org/10.1007/s00701-015-2649-4 